\title{
Embolic Stroke and Meningitis Secondary to Staphylococcus lugdunensis Native Valve Endocarditis
}

\author{
Wafa Ali AlDhaleei $\mathbb{D}^{1},{ }^{1}$ Akshaya Srikanth Bhagavathula $\mathbb{D}^{2},{ }^{2}$ and Rabia Aldoghaither ${ }^{3}$ \\ ${ }^{1}$ Internal Medicine Specialist, Sheikh Khalifa Medical City, P.O. Box: 51900, Abu Dhabi, UAE \\ ${ }^{2}$ Department of Internal Medicine, College of Medicine and Health Sciences, UAE University, P.O. Box: 15551, \\ Al Ain, UAE \\ ${ }^{3}$ Infectious Diseases Consultant, Sheikh Khalifa Medical City, P.O. Box: 51900, Abu Dhabi, UAE \\ Correspondence should be addressed to Wafa Ali AlDhaleei; wafa.ali.aldhaleei@gmail.com
}

Received 18 February 2019; Revised 1 April 2019; Accepted 16 April 2019; Published 28 April 2019

Academic Editor: Larry M. Bush

Copyright (c) 2019 Wafa Ali AlDhaleei et al. This is an open access article distributed under the Creative Commons Attribution License, which permits unrestricted use, distribution, and reproduction in any medium, provided the original work is properly cited.

Staphylococcus lugdunensis is a coagulase-negative staphylococcus that leads to destructive infective endocarditis. The clinical course of $S$. lugdunensis endocarditis is usually aggressive with a high mortality rate compared to endocarditis caused by other coagulase-negative staphylococcal species. Despite that, it is usually sensitive to Penicillin $G$, and surgical intervention is sometimes warranted. Here, we report a case of S. lugdunensis endocarditis complicated by both embolic stroke and meningitis.

\section{Introduction}

Staphylococcus lugdunensis is a Gram-positive, coagulasenegative Staphylococcus and was first described by Freney et al. in 1988 [1]. S. lugdunensis endocarditis is usually destructive in a native as well as a prosthetic valve. The incidence of $S$. lugdunensis endocarditis varies from 3 to 7 per 100,000 person-years annually worldwide [2]. S. lugdunensis meningitis has been reported in the previous literature as a single pathology [3-5]. However, it is worth mentioning that we report the first case of S. lugdunensis native valve endocarditis which was complicated by both embolic stroke and meningitis.

\section{The Case}

A 44-year-old nonalcoholic and nonsmoker man, known polymyositis, dyslipidemia, and asthma, presented to the hospital with fever for ten days, associated with a left-side headache, dry cough, and also night sweats in the past two months. He also reported impaired sensation over the left side of the face and body with left-sided facial droop and leftsided weakness. The patient reported a weight loss of $7 \mathrm{Kg}$ over the last two months. On the second day of fever, he was seen in a clinic and was given amoxicillin-clavulanic acid $1 \mathrm{~g}$ daily for seven days course, for possible otitis media. Despite this, his fever did not subside. Then, four days before the admission, he received cefixime $400 \mathrm{mg}$ (3 doses only) after which he was admitted.

Blood culture grew S. lugdunensis on the day of admission and day 3 of hospitalization, transthoracic echo (TTE) and transesophageal echo (TEE) identified vegetations on the aortic valve (reported to be $<1 \mathrm{~cm}$ ), and myxomatous thickened mitral valve (possible vegetations) with moderate mitral regurgitation (Figures 1 and 2). He was started on vancomycin for the treatment of infective endocarditis. His recent neurological deficits were attributed to embolic stroke secondary to the infective endocarditis. Repeat blood culture on the fifth day of hospitalization was negative. On the third day of hospitalization, the patient complained of worsening headache, and the CT brain showed diffuse leptomeningeal, tentorium, and falx enhancement, with highly suspicious faint linear enhancement along the right central sulcus. Lumbar puncture was done the following day, and the cerebrospinal fluid (CSF) analysis revealed leukocytosis of neutrophils predominance of $47 \%$ with very high protein and very low glucose levels. Moreover, CSF culture grew S. lugdunensis. The patient received 


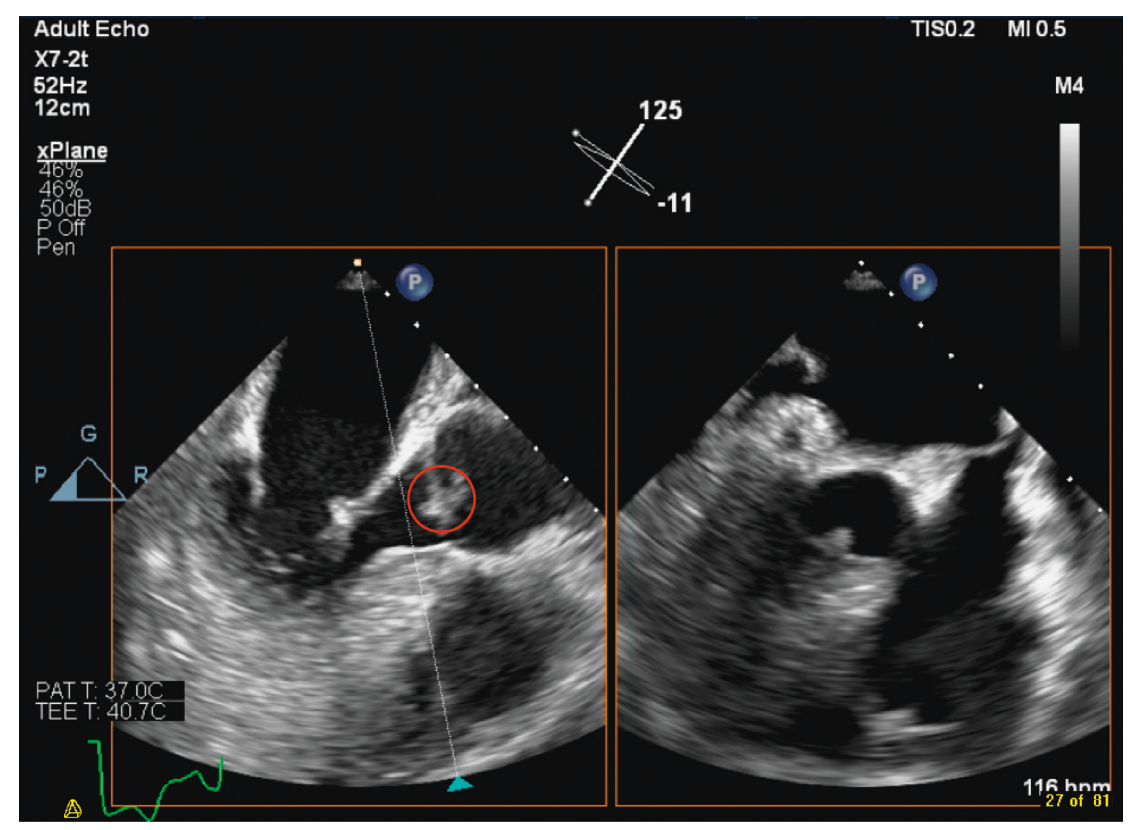

FIgURE 1: Aortic valve vegetation.

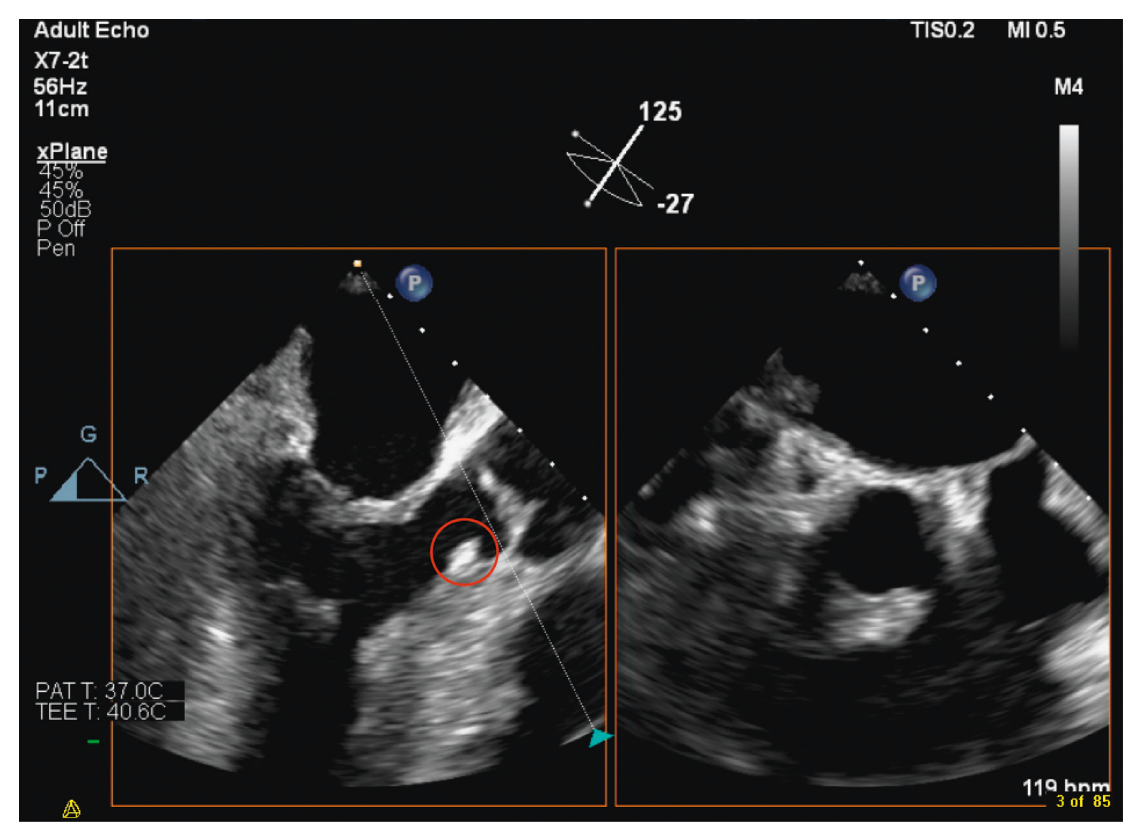

FIgURE 2: Subaortic valve vegetation.

four days of vancomycin alone (loading dose of $1 \mathrm{~g}$ then adjusted according to the vancomycin trough level). Then, flucloxacillin $2 \mathrm{~g} \mathrm{q} 6 \mathrm{hrs}$ was added to vancomycin for a total duration of 17 days. After that, the patient received flucloxacillin $2 \mathrm{~g}$ q6 hrs alone for an additional 27 days. Flucloxacillin was added to vancomycin, given the severity of the case presentation. The cardiothoracic team evaluated the patient, and they decided that surgical intervention is not indicated. Repeated TEE before discharge revealed aortic valve vegetation which is smaller in size (Figure 3). Upon discharge, he was able to ambulate with support and required outpatient rehabilitation follow-ups.

\section{Discussion}

We report the first case of $S$. lugdunensis endocarditis causing meningitis and embolic stroke. S. lugdunensis was first described in 1988 as a causative organism for endocarditis [6]. It can produce biofilms that enable it to adhere to prosthetic materials and native tissues [7]. S. lugdunensis endocarditis has also been reported in patients with cardiac implantable devices and prosthetic valves [8-11].

S. lugdunensis native valve endocarditis has been associated with various complications ranging from a myocardial abscess, septic emboli, and valve perforation [11]. Our 


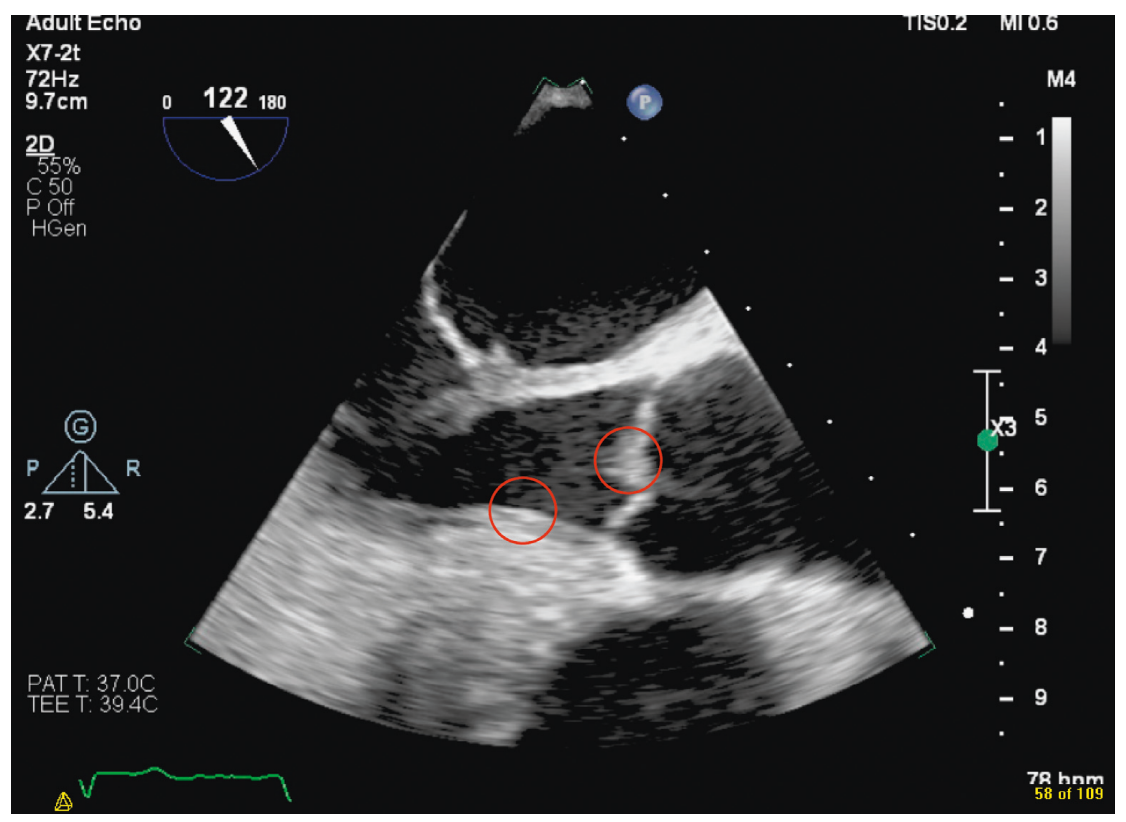

Figure 3: Aortic valve posttreatment.

patient had involvement of the aortic valve, but other valves such as mitral and tricuspid were also involved but to a lesser extent. Ishiekwene et al. also reported the involvement of the aortic valve with a ventricular septal defect [7]. However, Chung et al. reported the involvement of the tricuspid valve [12]. Kline et al. reported case series of different valve involvement [13].

The neurological manifestations secondary to septic embolization can range from altered sensation, weakness, and visual defects [14]. It appears that embolic stroke secondary to $S$. lugdunensis endocarditis is highly seen in middle-aged men and women [2]. However, S. lugdunensis meningitis has been reported in the previous literature as a complication postoperative and a single pathology without endocarditis [3-5]. Our patient presented with endocarditis complicated by both stroke and meningitis, in which meningitis has not been yet reported as a complication of $S$. lugdunensis endocarditis.

S. lugdunensis endocarditis has been associated with high mortality [15], although most of the S. lugdunensis isolates are sensitive to Penicillin G $[13,16]$. High mortality is associated with the ability of the $S$. lugdunensis to cause tissue destruction leading to acute heart failure. Therefore, early surgical intervention has been associated with favorable outcomes in such cases $[6,12]$. The efficacy of medical treatment versus a combination of medical and surgical treatment needs further investigation [2]. Our patient responded to medical treatment alone.

\section{Conclusion}

S. lugdunensis endocarditis is unusual. We reported a case of S. lugdunensis endocarditis complicated by both embolic stroke and meningitis. Careful evaluation and assessment are warranted to direct the appropriate therapy of patients.

\section{Conflicts of Interest}

The authors declare no conflicts of interest in this study.

\section{Acknowledgments}

We thank our colleague Ahlam Alawadhi, Cardiology Fellow, for retrieving the images of the TEE.

\section{References}

[1] J. Freney, Y. Brun, M. Bes et al., "Staphylococcus lugdunensis sp. nov. and Staphylococcus schleiferi sp. nov., two species from human clinical specimens," International Journal of Systematic Bacteriology, vol. 38, no. 2, pp. 168-172, 1988.

[2] H. Kyaw, F. Raju, A. Shaikh et al., "Staphylococcus lugdunensis endocarditis and cerebrovascular accident: a systemic review of risk factors and clinical outcome," Cureus, vol. 10, no. 4, article e2469, 2018.

[3] N. Kaabia, D. Scauarda, G. Lena, and M. Drancourt, "Molecular identification of Staphylococcus lugdunensis in a patient with meningitis," Journal of Clinical Microbiology, vol. 40, no. 5, pp. 1824-1825, 2002.

[4] Y. Sasaki, A. Kanamaru, M. H. Uchida, H. Yano, and H. Tada, "A case of bacterial meningitis caused by methicillin-resistant Staphylococcus lugdunensis after surgery," Rinsho Shinkeigaku, vol. 56, no. 11, pp. 773-776, 2016.

[5] T. Spanu, D. Rigante, G. Tamburrini et al., "Ventriculitis due to Staphylococcus lugdunensis: two case reports," Journal of Medical Case Reports, vol. 2, no. 1, p. 267, 2008.

[6] A. Renzulli, A. Della Corte, M. Torella, G. Dialetto, and M. Cotrufo, "Mitral and aortic valve endocarditis due to Staphylococcus lugdunensis," Texas Heart Institute Journal, vol. 27, no. 1, pp. 67-69, 2000.

[7] C. Ishiekwene, M. Ghitan, M. Kuhn-Basti, E. Chapnick, and Y. S. Lin, "Staphylococcus lugdunensis endocarditis with destruction of the ventricular septum and multiple native valves," IDCases, vol. 7, pp. 14-15, 2017. 
[8] Y.-T. Tsao, W.-J. Wang, S.-W. Lee, J.-C. Hsu, F.-M. Ho, and W.-L. Chen, "Characterization of Staphylococcus lugdunensis endocarditis in patients with cardiac implantable electronic devices," International Journal of Infectious Diseases, vol. 16, no. 6, pp. e464-e467, 2012.

[9] A. Chopra, D. Gulati, N. Woldenberg, and M. Singh, "Intracardiac lead endocarditis due to Staphylococcus lugdunensis," International Journal of Infectious Diseases, vol. 14, pp. e291-e293, 2010.

[10] M. Ward and K. M. Boehm, "Pacemaker related infective endocarditis from Staphylococcus lugdunensis: a case report," Case Reports in Critical Care, vol. 2013, Article ID 180401, 3 pages, 2013.

[11] I. Anguera, "Staphylococcus lugdunensis infective endocarditis: description of 10 cases and analysis of native valve, prosthetic valve, and pacemaker lead endocarditis clinical profiles," Heart, vol. 91, no. 2, p. e10, 2005.

[12] K.-P. Chung, H.-T. Chang, C.-H. Liao, F.-Y. Chu, and P.-R. Hsueh, "Staphylococcus lugdunensis endocarditis with isolated tricuspid valve involvement," Journal of Microbiology, Immunology and Infection, vol. 45, no. 3, pp. 248-250, 2012.

[13] J. A. Kline, T. J. Byrnes, and J. P. Myers, "Staphylococcus lugdunensis: the "lyon heart" pathogen report of 5 cases of endocarditis and review of the adult literature," Infectious Diseases in Clinical Practice, vol. 25, no. 3, pp. 134-146, 2017.

[14] M. David, M. Loftsgaarden, and F. Chukwudelunzu, "Embolic stroke caused by Staphylococcus lugdunensis endocarditis complicating vasectomy in a 36-year-old man," Texas Heart Institute Journal, vol. 42, no. 6, pp. 585-587, 2015.

[15] J.-F. Lin, C.-W. Cheng, A.-J. Kuo et al., "Clinical experience and microbiologic characteristics of invasive Staphylococcus lugdunensis infection in a tertiary center in northern Taiwan," Journal of Microbiology, Immunology and Infection, vol. 48, no. 4, pp. 406-412, 2015.

[16] H. Schandiz, N. Olav Hermansen, T. Jørgensen, and B. Roald, "Staphylococcus lugdunensis endocarditis following vasectomyreport of a case history and review of the literature," APMIS, vol. 123, no. 8, pp. 726-729, 2015. 


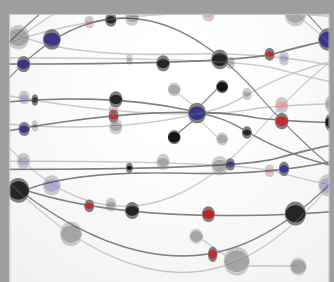

The Scientific World Journal
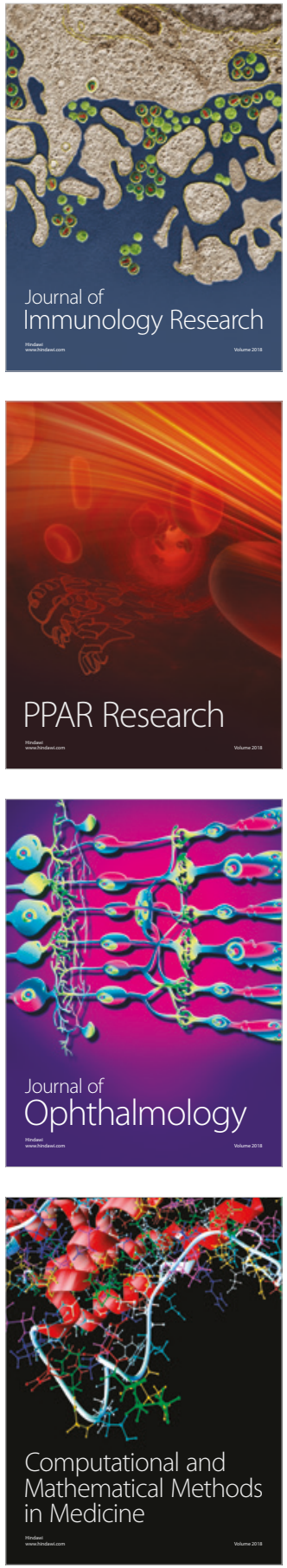

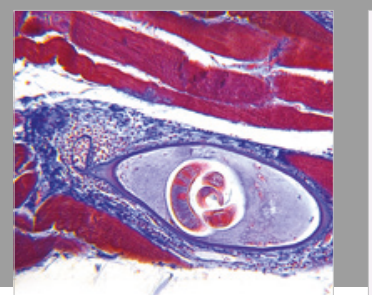

Gastroenterology Research and Practice

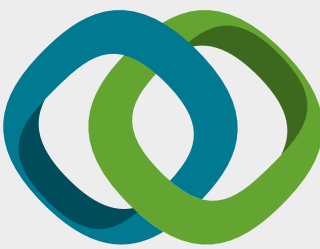

\section{Hindawi}

Submit your manuscripts at

www.hindawi.com
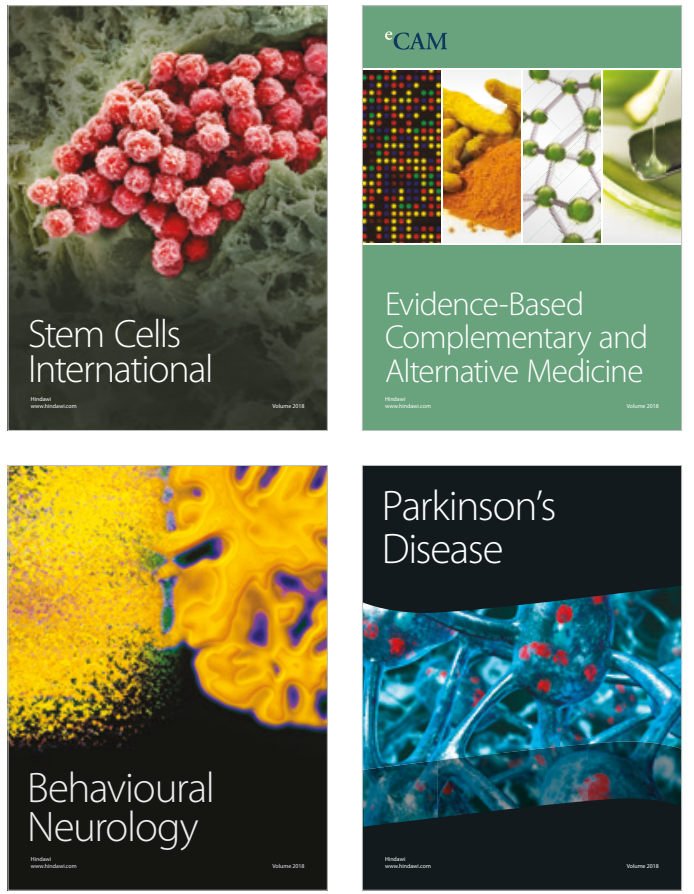

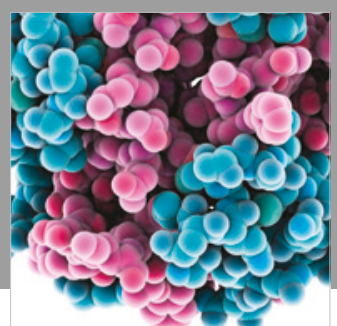

ournal of

Diabetes Research

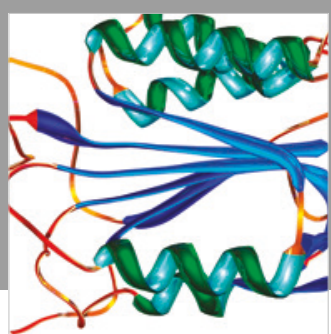

Disease Markers
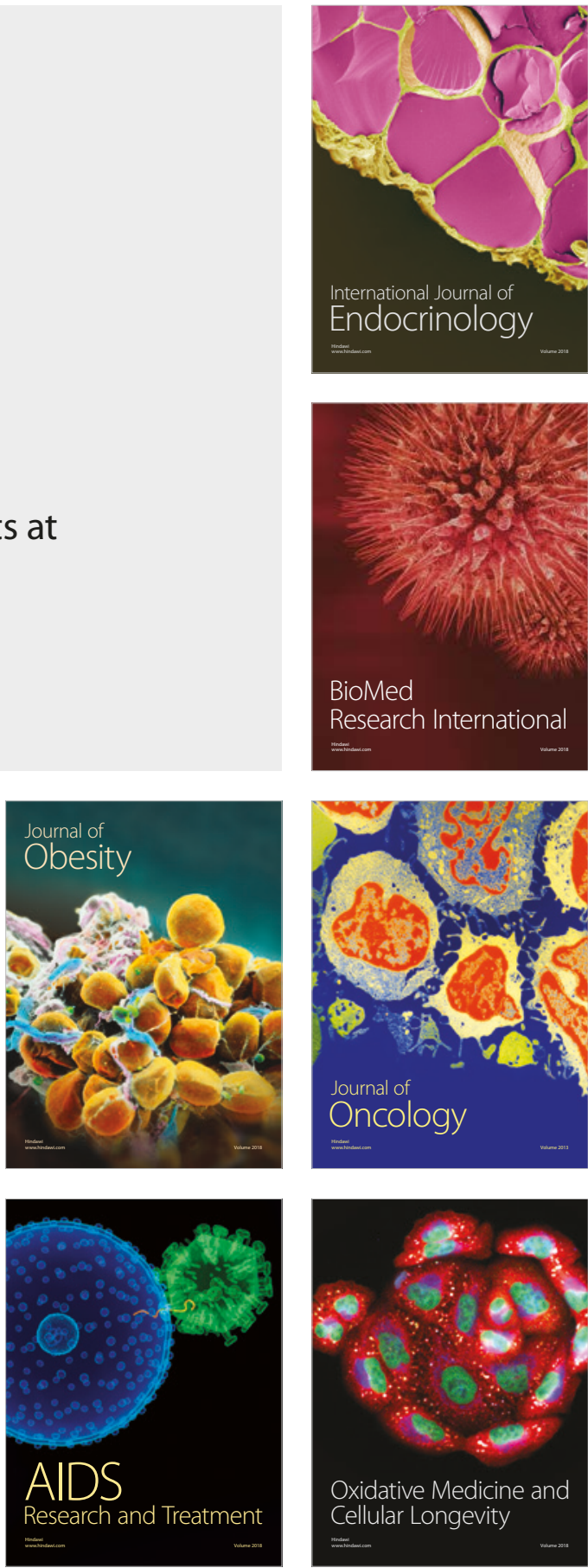